\title{
Role of gap junction intercellular communication in testicular leydig cell apoptosis induced by oxaliplatin via the mitochondrial pathway
}

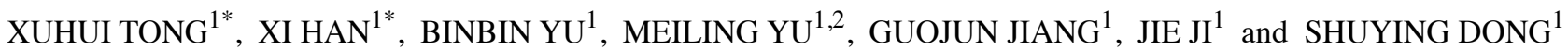 \\ ${ }^{1}$ Faculty of Pharmacy, Bengbu Medical College, Bengbu, Anhui 233030; ${ }^{2}$ Department of Pharmacy, \\ The First Affiliated Hospital of Bengbu Medical College, Bengbu, Anhui 233004, P.R. China
}

Received August 5, 2014; Accepted October 17, 2014

DOI: $10.3892 /$ or.2014.3571

\begin{abstract}
Platinum agents are widely used in the chemotherapy of testicular cancer. However, adverse reactions and resistance to such agents have limited their application in antineoplastic treatment. The aim of the present study was to determine the role of gap junction intercellular communication (GJIC) composed of $\mathrm{Cx} 43$ on oxaliplatin-induced survival/apoptosis in mouse leydig normal and cancer cells using MTT, Annexin V/ PI double staining assays and western blot analysis. The results showed that GJIC exerted opposite effects on the mouse leydig cancer (I-10) and normal $\left(\mathrm{TM}_{3}\right)$ cell apoptosis induced by oxaliplatin. In leydig cancer cells, survival of cells exposed to oxaliplatin was substantially reduced when gap junctions formed as compared to no gap junctions. Pharmacological inhibition of gap junctions by oleamide and 18- $\alpha$-glycyrrhetinic acid resulted in enhanced survival/decreased apoptosis while enhancement of gap junctions by retinoic acid led to decreased survival/increased apoptosis. These effects occurred only in high-density cultures (gap junction formed), while the pharmacological modulations had no effects when there was no opportunity for gap junction formation. Notably, GJIC played an opposite (protective) role in normal leydig cells survival/ apoptosis following exposure to oxaliplatin. Furthermore, this converse oxaliplatin-inducing apoptosis exerted through the functional gap junction was correlated with the mitochondrial pathway-related protein $\mathrm{Bcl}-2 / \mathrm{Bax}$ and caspase-3/9. These results suggested that in testicular leydig normal/cancer cells, GJIC plays an opposite role in oxaliplatin-induced apoptosis via the mitochondrial pathway.
\end{abstract}

Correspondence to: Dr Shuying Dong, Faculty of Pharmacy, Bengbu Medical College, 2600 Donghai Road, Bengbu, Anhui 233030, P.R. China

E-mail: bbmcdsy@126.com

${ }^{*}$ Contritubed equally

Key words: oxaliplatin, gap junction, apoptosis, testis

\section{Introduction}

Leydig cells are adjacent to the seminiferous tubules in the testicle. Leydig cells release a variety of androgens, including testosterone, androstenedione and dehydroepiandrosterone, when stimulated by the luteinizing hormone $(1,2)$. When Leydig cells grow in an uncontrolled manner, they may form Leydig cell tumors. For testicular cancer, platinum agents are considered important and superior candidates in chemotherapy. Cisplatin is the most common platinum agent. However, its continued use is greatly limited by severe adverse reactions (nephrotoxicity and neurotoxicity) and intrinsic or acquired drug resistance $(3,4)$. Oxaliplatin, the third-generation platinum drug, has been developed to overcome cisplatin resistance in various types of cancer (5-9). However, patients receiving oxaliplatin are affected by sensory neuropathy and neurotoxicity. Thus, identifying the factors that increase the sensitivity of cancer cells to oxaliplatin and decrease the cytotoxicity in normal cells is crucial.

Gap junctions are the intercellular channels that allow direct intercellular exchange of ions, nutrients and small signaling molecules $(<1 \mathrm{kDa})$. Gap junction channels comprise connexins, the membrane proteins encoded by a multi-gene family $(10,11)$. At present, 21 connexins have been identified in human, five of which are present in testis tissues, whereas only $\mathrm{Cx} 43$ is localized in the membrane appositions between leydig cells $(11,12)$. In testis, Cx43 is pivotal for the development of spermatogenesis, and deficiency of Cx43 may lead to male infertility $(13,14)$.

Findings of recent studies have shown that gap junctional intercellular communication (GJIC) can increase the cytotoxicity after antineoplastic drug treatment, gene therapy or radiotherapy (15-19). The phenomenon was first described in the herpes simplex virus-thymidine kinase/ganciclovir (HSVtk/GCV) approach to tumor gene therapy and is associated with the 'bystander effect' (20). In the increased cytotoxicity mediated though GJIC, some death signals and/or toxic drug metabolites filtered into the adjacent cells. However, GJIC also produce the protective effects in certain normal cells such as epithelia, astrocyte and neuron, after insults including oxidative stress and ischemia (21-25). Previously, we showed opposite survival after cisplatin treatment in $\mathrm{TM}_{3}$, 
$\mathrm{TM}_{4}$ testicular normal cells and MLTC-1 cancer cells (26). However, the mechanisms of leydig normal/cancer cell survival discrepancy, particularly oxaliplatin-induced apoptosis in the oncogenic status of cell types remains to be elucidated. The aim of the present study was to determine the role of GJIC composed of $\mathrm{Cx} 43$ on oxaliplatin-induced survival/apoptosis in mouse leydig normal and cancer cells.

\section{Materials and methods}

Materials. Primary antibodies for this study included: anti-Cx43 which was purchased from Sigma-Aldrich (St. Louis, MO, USA); anti-Bcl-2 and anti-Bax from Santa Cruz Biotechnology, Inc. (Dallas, TX, USA); anti-caspase-9 from Proteintech (Chicago, IL, USA) and anti-caspase-3 from Abcam (Cambridge, MA, USA). Oxaliplatin, 3-[4,5-dimethylthiazol-2-yl]-2,5-diphenyltetrazolium bromide (MTT), retinoic acid (RA), 18- $\alpha$-glycyrrhetinic acid (18- $\alpha$-GA) and oleamide (Olea) were purchased from Sigma-Aldrich. The FITC Annexin V/Dead Cell Apoptosis kit with fluorescein isothiocyanate (FITC) Annexin V and propidium iodide (PI), DMEM/F-12, F12, fetal bovine serum, horse serum and calcein-AM were purchased from Invitrogen (Grand Island, NY, USA). Secondary antibodies for western blotting were purchased from Amersham Biosciences Corp. (Piscataway, NJ, USA). The BCA protein assay kit was purchased from Pierce Biotechnology (Rockford, IL, USA). All other reagents were obtained from Sigma-Aldrich.

Cell lines and cell culture. The mouse leydig cell line $\left(\mathrm{TM}_{3}\right)$ and mouse leydig cancer cell line (I-10) were obtained from the American Type Culture Collection (Manassas, VA, USA). $\mathrm{TM}_{3}$ cells were cultured in DMEM/F12 supplemented with $5 \%$ horse serum and $2.5 \%$ fetal bovine serum. I-10 cells were cultured in F12 supplemented with 15\% horse serum and 2.5\% fetal bovine serum. The cells were grown at $37^{\circ} \mathrm{C}$ in an atmosphere of $5 \% \mathrm{CO}_{2}$ in air.

Modulation of GJIC. The pharmacological drugs for GJIC modulation were dissolved in dimethylsulfoxide (DMSO) as stock solutions (16). For the potentiation process, the cells were incubated with GJ potentiator retinoic acid $(10 \mu \mathrm{M}$ in DMSO) $24 \mathrm{~h}$ prior to oxaliplatin exposure and during the oxaliplatin treatment. For inhibition, the cells were incubated with the $18-\alpha-\mathrm{GA}(10 \mu \mathrm{M}$ in DMSO) and oleamide ( $25 \mu \mathrm{M}$ in DMSO) GJ inhibitors $1 \mathrm{~h}$ prior to exposure to oxaliplain and during the oxaliplatin treatment. Control cells were incubated with DMSO alone.

Cell survival following oxaliplatin treatment. Oxaliplatin stock solutions were freshly prepared at $10 \mathrm{mM}$ in phosphate-buffered solution (PBS). Cell exposure to oxaliplatin was performed in the dark. Cell survival after exposure to oxaliplatin was determined using the MTT assay. To observe the role of GJIC in oxaliplatin cytotoxicity, the cells were seeded at high $\left(25,000 / \mathrm{cm}^{2}\right)$ or low density $\left(100 / \mathrm{cm}^{2}\right)$ in 96 -well plates and incubated at $37^{\circ} \mathrm{C}$ as previously described (16). The high- or low-density cells were then treated with $10 \mu \mathrm{M}$ oxaliplatin for $24 \mathrm{~h}$. The medium was removed and the cells were incubated with $0.5 \mathrm{mg} / \mathrm{ml}$ MTT solution for $4 \mathrm{~h}$. The absorbance (optical density, OD) at $570 \mathrm{~nm}$ was measured using a microplate reader (Bio-Rad Laboratories, Hercules, CA, USA). The rate of the cell surviving fraction was determined using the formula:

$$
\text { Surviving fraction }=\frac{\text { OD oxaliplatin }}{\text { OD control }}
$$

'Parachute' dye-coupling assay. The gap junction function assay was performed as previously described $(27,28)$. Donor and receiver cells were grown to confluence in 12-well plates. Donor cells from one well were incubated with a freshly prepared solution of $10 \mu \mathrm{g} / \mathrm{ml}$ calcein AM in growth medium for $30 \mathrm{~min}$ at $37^{\circ} \mathrm{C}$. In the live cells, calcein AM was converted intracellularly into the gap junction-permeable dye calcein. The donor cells were then trypsinized and seeded in the receiver cells at a 1:200 donor/receiver ratio. The cells were allowed to adhere to the monolayer of the receiver cells and form gap junctions for $4 \mathrm{~h}$ at $37^{\circ} \mathrm{C}$, and then examined using a fluorescence microscope (Olympus CK40; Olympus, Tokyo, Japan). The average number of receiver cells containing dye per donor cell was counted and normalized to that of control cultures.

Annexin V/PI double staining assay. Cells $\left(5 \times 10^{4}\right)$ were plated in 6-well dishes and cultured for $48 \mathrm{~h}$ (80-100\% confluence). The cells were treated with $20 \mu \mathrm{M}$ oxaliplatin, incubated for $12 \mathrm{~h}$, collected and rinsed twice with PBS. The cells were resuspended in binding buffer and incubated with FITC-conjugated Annexin $\mathrm{V}$ and propidium iodide (PI) for $15 \mathrm{~min}$ at room temperature in the dark using the FITC/Annexin-V apoptosis detection kit. The samples were immediately analyzed on an Auccri C6 flow cytometer (BD Biosciences, San Jose, CA, USA). Early apoptotic cells were shown in the lower right (Annexin V+/PI-) quadrant.

Western blot analysis. Cells were seeded in 6-well plates and treated as described above. The cells were washed three times with cold PBS and collected using lysis buffer. The cell lysate was sonicated and centrifuged at $12,000 \mathrm{rpm}$ for $30 \mathrm{~min}$ at $4^{\circ} \mathrm{C}$. Protein concentration was determined using the BCA protein assay kit. Protein $(50 \mu \mathrm{g})$ from each sample was analyzed by SDS-PAGE followed by immunoblotting. Antibodies against Cx43 (1:4,000), Bcl-2 (1:200), Bax (1:200), caspase-3 (1:1,000), caspase-9 (1:500) and $\beta$-actin $(1: 10,000)$ were used. Western blots bands were defined in the linear range of detection and the intensities of the resulting bands were quantified using Quantity One software on a GS-800 densitometer (Bio-Rad Laboratories, Hercules, CA, USA).

Statistical analysis. The experiments had a minimum of three determinations. The data were expressed as means \pm standard deviation (SD). Statistical significance $(\mathrm{P}<0.05)$ was determined with one-way ANOVA.

\section{Results}

Effect of cell density on the cytotoxicity of oxaliplatin. Gap junctions are formed by the adjacent cells expressing connexin hemichannels and GJIC can take effect only when 

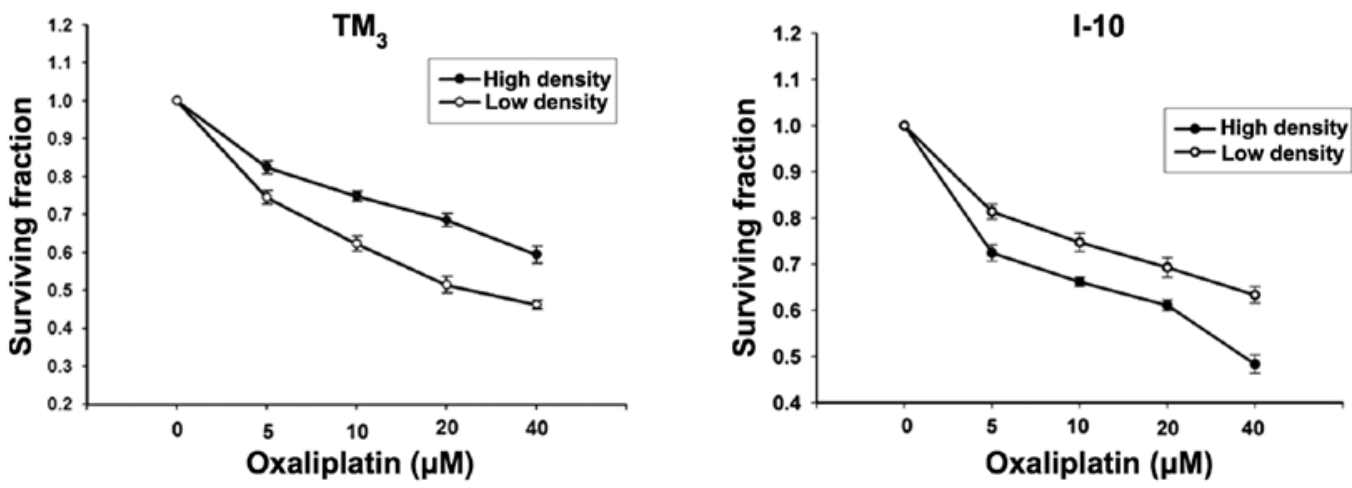

Figure 1. Effect of oxaliplatin on cell survival of mouse leydig normal and cancer cells. The survival of leydig normal TM $\mathrm{T}_{3}$ cells and I-10 cancer cells were treated with oxaliplatin at concentrations of $0-40 \mu \mathrm{M}$ at a high-cell $\left(25,000\right.$ cells $\left./ \mathrm{cm}^{2}\right)$ or low-cell $\left(100\right.$ cells $\left./ \mathrm{cm}^{2}\right)$ density. Data points are means \pm SD for five experiments.
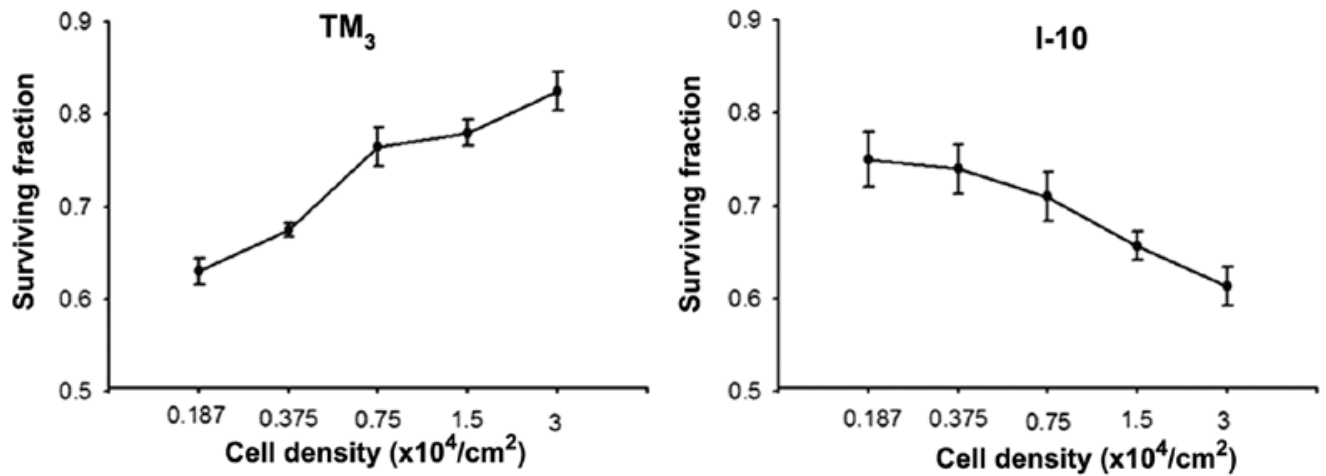

Figure 2. Survival of cells treated with oxaliplatin is modulated by cell density. Survival of cells treated with $10 \mu \mathrm{M}$ oxaliplatin at a cell density of $\left(0.187-3 \times 10^{4} / \mathrm{cm}^{2}\right)$ as assayed by MTT. Data points are mean \pm SD for five experiments.

cells are able to communicate with each other. To determine the effect of GJIC on cell viability, mouse leydig normal cells $\left(\mathrm{TM}_{3}\right)$ and cancer cells (I-10) were seeded under conditions where gap junction formation was possible (high density, 25,000 cells $/ \mathrm{cm}^{2}$ ) or not possible (low density, 100 cells $/ \mathrm{cm}^{2}$ ). Cytotoxicity following oxaliplatin treatment was determined using an MTT assay. Fig. 1 shows the cell survival subsequent to treatment with oxaliplatin for $24 \mathrm{~h}$ at a high (GJ formed) and low density (no GJ formed). Cell survival at a high or low density decreased as the concentration of oxaliplatin increased from 5 to $40 \mu \mathrm{M}$. However, cell survival in culture was opposite in leydig normal cells $\left(\mathrm{TM}_{3}\right)$ and cancer cells (I-10): at all concentrations of oxaliplatin, the surviving fraction of $\mathrm{TM}_{3}$ cells at a high density was substantially greater than that at low density. However, the surviving fraction of I-10 cells at a high density was substantially less than that at low density.

Fig. 2 shows the effect of cell seeding density on the oxaliplatin cytotoxicity in $\mathrm{TM}_{3}$ and I-10 cells. After 48-h incubation, the two cells seeded at the highest density reached $80-100 \%$ confluence. In mouse testicular leydig $\mathrm{TM}_{3}$ cells treated with $10 \mu \mathrm{M}$ oxaliplatin for $24 \mathrm{~h}$, the surviving fraction increased from $0.63 \pm 0.01$ to $0.82 \pm 0.02$. By contrast, the surviving fraction decreased from $0.75 \pm 0.03$ to $0.61 \pm 0.02$ in cancer I-10 cells.

These results demonstrated that when at high density oxaliplatin toxicity is decreased in the testicular normal cells, whereas toxicity is increased in the cancer cells, suggesting that the effects of high-cell density may be due to GJIC.

Effect of GJIC on cell viability following oxaliplatin treatment. Based on the results described above, we investigated the role of GJIC on the toxicity of oxaliplatin and further confirmed the effect of high-cell density is due to GJIC. Gap junction functions were manipulated in two ways: inhibition of junctional channels by $18-\alpha-\mathrm{GA}$ and oleamide, and enhancement of junctional channels by retinoic acid.

Retinoic acid (RA) is a common agent that increases the GJIC in numerous cells $(16,29)$. The 'Parachute' dye spread assay was used to determine the effect of RA on the GJIC of the leydig normal $\left(\mathrm{TM}_{3}\right)$ and cancer cells (I-10). Fig. 3A shows that treatment with $10 \mu \mathrm{M}$ RA for $24 \mathrm{~h}$ increased GJIC in the $\mathrm{TM}_{3}$ and I-10 cells. In the low-density cell cultures (no GJ formed), RA did not affect the surviving fraction of cells. However, for $\mathrm{TM}_{3}$ cells cultured in high density, RA increased the oxaliplatin surviving fraction, yielding substantially decreased cytotoxicity (Fig. 3B). Notably, for I-10 cells cultured in high density, RA clearly increased cytotoxicity. Thus, the GJ potentiator (RA) increased/decreased leydig normal/cancer cell survival only when the functional gap junction was formed (high cell density).

18- $\alpha$-GA and oleamide (olea) are widely used to inhibit GJIC in different cells $(16,30)$. In testicular normal and cancer cells, treatment with $10 \mu \mathrm{M} 118-\alpha-\mathrm{GA}$ and $25 \mu \mathrm{M}$ oleamide 
$\mathrm{TM}_{3}$

A

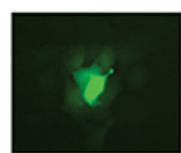

control

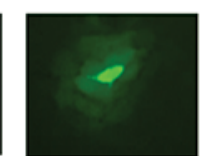

RA

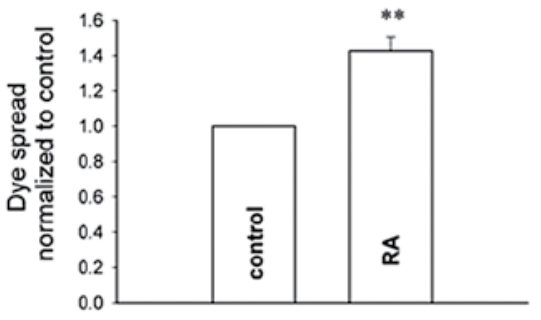

B

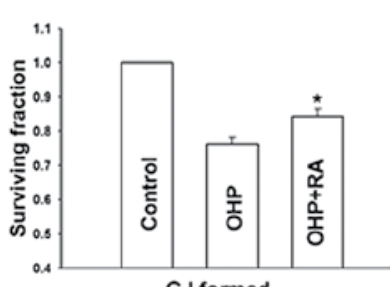

GJ formed

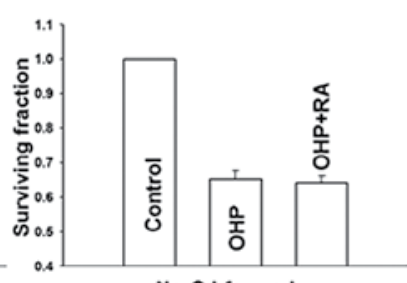

No GJ formed

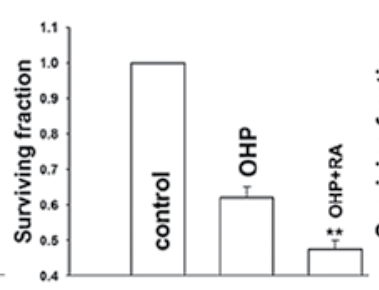

GJ formed
I-10

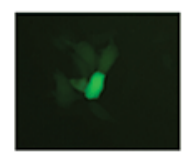

control

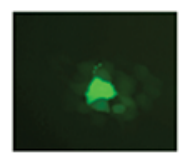

RA

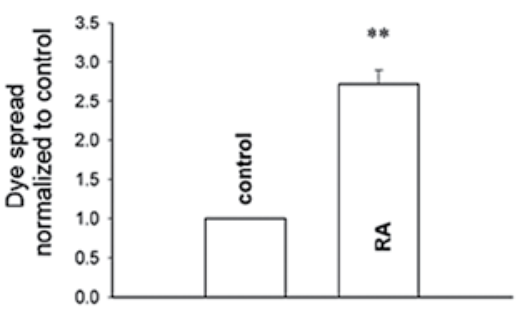

Figure 3. Effect of GJ potentiator (retinoic acid) on the survival of cells treated with oxaliplatin. (A) Fluorescence image of the dye coupling through gap junctions in $\mathrm{TM}_{3}$ (left panel) and I-10 cells (right panel) as shown by the parachute dye coupling assay. RA (10 $\left.\mu \mathrm{M}, 24 \mathrm{~h}\right)$ can increase the dye coupling. Columns are mean from five independent experiments; bars are the standard deviation. ${ }^{* *} \mathrm{P}<0.01$, significantly different from the control group. (B) Effects of RA on the survival of cells treated with oxaliplatin $(10 \mu \mathrm{M}, 24 \mathrm{~h})$ in the high density (GJ formed) and low density (no GJ formed) groups. Columns are means from five independent experiments; bars are the standard deviation. ${ }^{*} \mathrm{P}<0.05$ and ${ }^{* *} \mathrm{P}<0.01$, significantly different from oxaliplatin group. RA, retinoic acid; OHP, oxaliplatin.

$\mathrm{TM}_{3}$

A

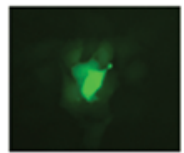

control

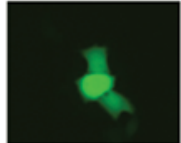

18- $\alpha-G A$

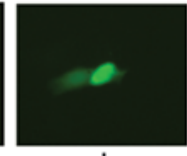

olea

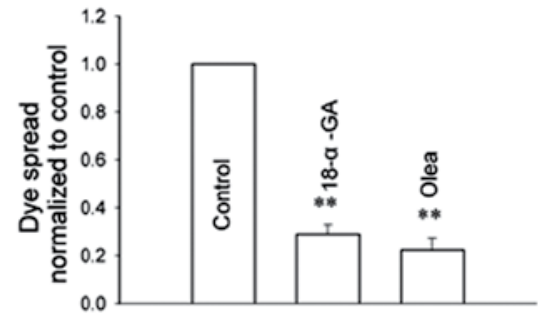

B

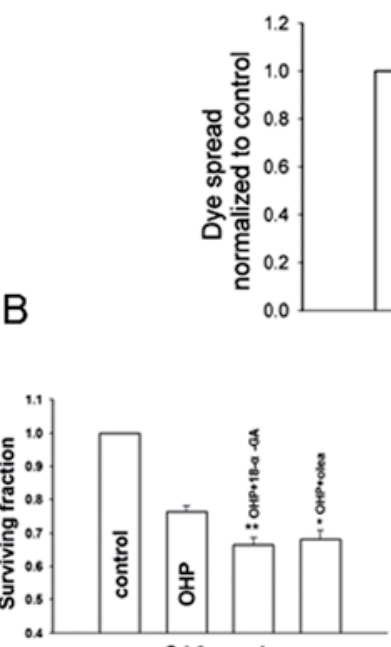

GJ formed

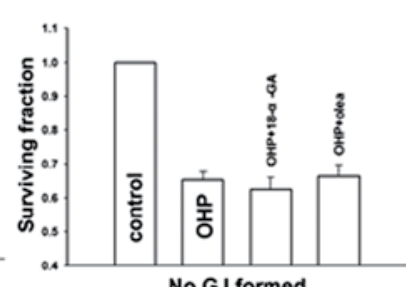

No GJ formed

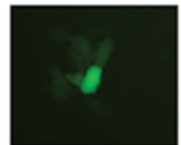

control

\section{$\mathrm{I}-10$}

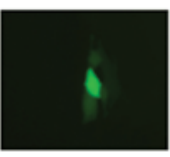

18-a-GA

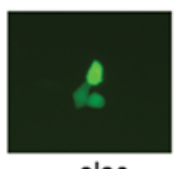

olea

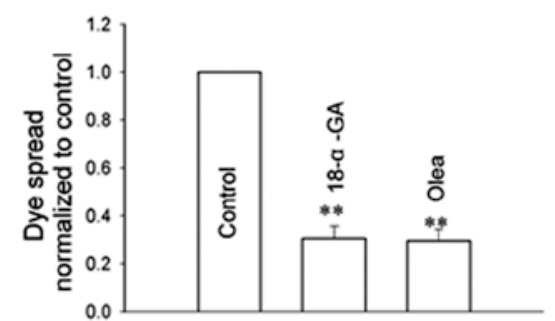

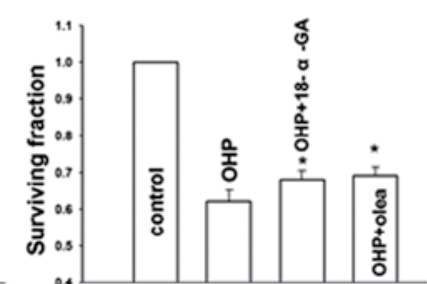

GJ formed

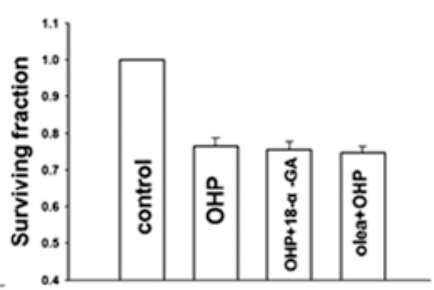

No GJ formed

Figure 4. Effect of GJ inhibitors (18- $\alpha$-GA and oleamide) on the survival of cells treated with oxaliplatin. (A) Fluorescence image of the dye coupling through gap junctions in $\mathrm{TM}_{3}$ (left panel) and I-10 cells (right panel) as shown by parachute dye coupling assay. 18- $\alpha$-GA $(10 \mu \mathrm{M}, 1 \mathrm{~h})$ and oleamide (25 $\left.\mu \mathrm{M}, 1 \mathrm{~h}\right)$ inhibit the dye coupling significantly. Columns are the mean from five independent experiments; bars are standard deviation. ${ }^{* *} \mathrm{P}<0.01$, significantly different from the control group. (B) Effects of 18- $\alpha-\mathrm{GA}$ and oleamide on the survival of cells treated with oxaliplatin $(10 \mu \mathrm{M}, 24 \mathrm{~h})$ in the high-density (GJ formed) and low-density (no GJ formed) groups. Columns are the means from five independent experiments; bars are the standard deviation. ${ }^{*} \mathrm{P}<0.05$ and ${ }^{* * *} \mathrm{P}<0.01$, significantly different from the oxaliplatin group. Olea, oleamide; OHP, oxaliplain. 

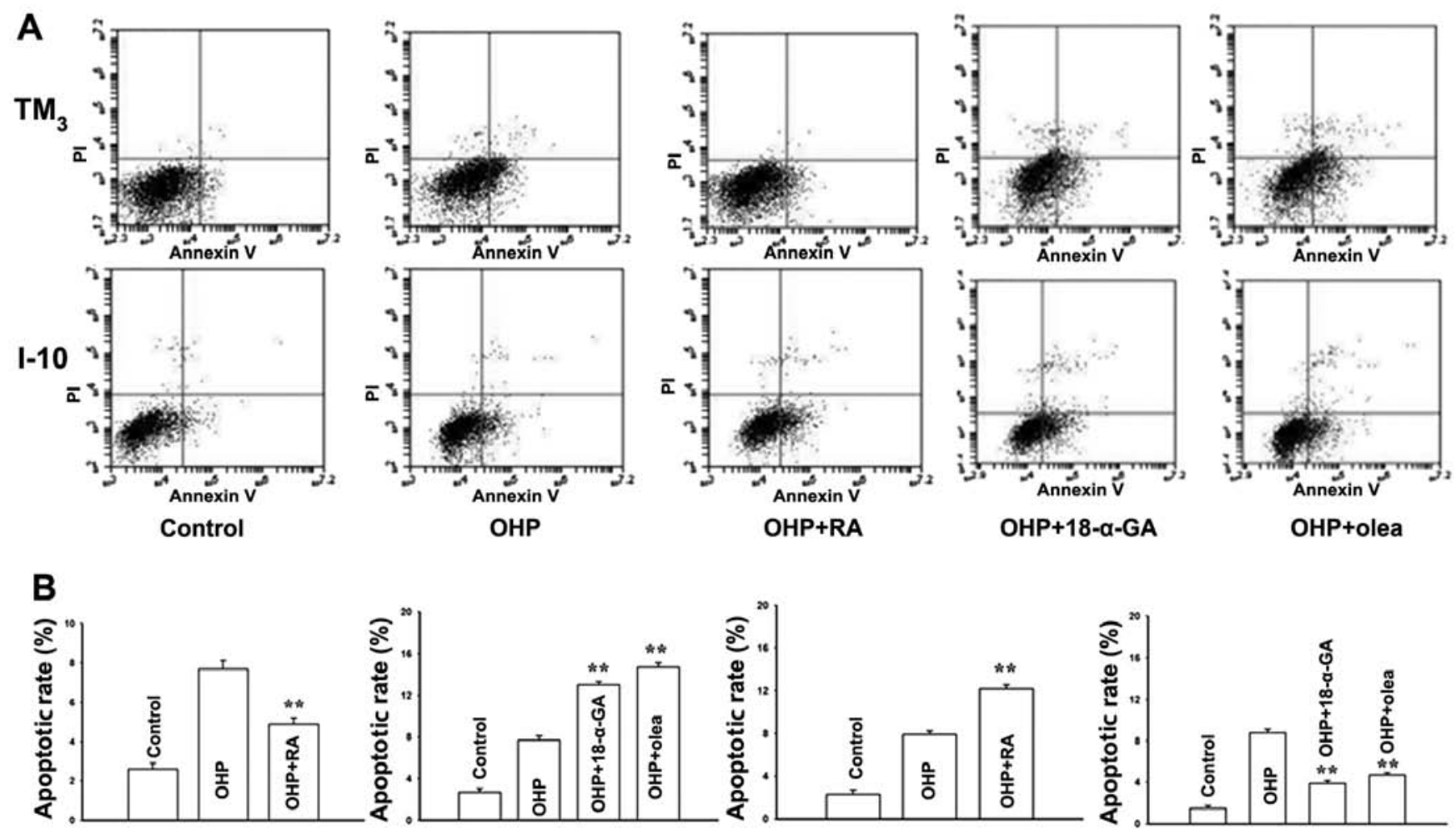

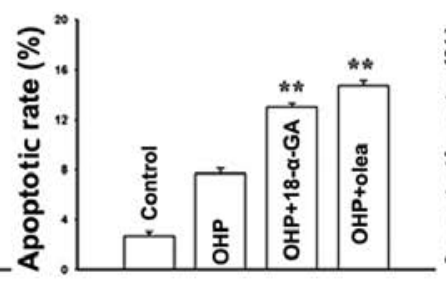

$\mathrm{TM}_{3}$
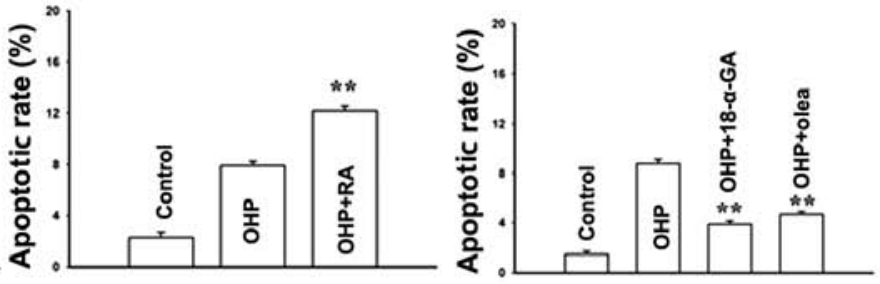

I-10

Figure 5. Effect of gap junction on apoptosis induced with cisplatin as determined by flow cytometry. (A) The apoptotic rate treated with $20 \mu \mathrm{M}$ oxaliplatin for $12 \mathrm{~h}$ was determined by flow cytometry labeled with Annexin V/PI. The percentage of cells positive for only Annexin V is indicated in the lower right quadrants and that of cells positive for Annexin V and PI is indicated in the top right quadrants. (B) Effect of gap junction modulator (potentiator, RA; inhibitor, 18- $\alpha$-GA and oleamide) on the apoptotic rates. Columns are the means from three independent experiments; bars are the standard deviation. ${ }^{* *} \mathrm{P}<0.01$, significantly different from the oxaliplatin group. OHP, oxaliplatin; RA, retinoic acid; Olea, oleamide.

for $1 \mathrm{~h}$ can decrease GJIC (Fig. 4A). In the low-density cell cultures (no GJ formed), the 18- $\alpha$-GA and oleamide GJIC inhibitors did not affect oxaliplatin cytotoxicity (Fig. 4B). In high-density $\mathrm{TM}_{3}$ cell cultures, the two inhibitors increased oxaliplatin cytotoxicity. However, for the high-density I-10 cell cultures, 18- $\alpha-\mathrm{GA}$ and oleamide increased surviving fraction. Thus, GJ inhibitor (18- $\alpha$-GA, oleamide) decreased/increased leydig normal/cancer cell survival only when the functional gap junction was formed (high cell density).

Therefore, the data indicated that the density dependence of oxaliplatin responses was mediated through GJIC. Furthermore, the oxaliplatin responses exerted an opposite effect in mouse leydig normal and cancer cells.

Effect of GJIC on the oxaliplatin-induced apoptosis. Apoptosis plays a vital role in tissue development and homeostasis and is involved in the oxaliplatin antineoplastic effect (31). Annexin V and PI double staining were used to assess the effect of GJIC on oxaliplatin-induced apoptosis. Fig. 5 shows early apoptosis induced with $20 \mu \mathrm{M}$ oxaliplatin for $12 \mathrm{~h}$ in leydig normal $\mathrm{TM}_{3}$ and I-10 cancer cells.

Fig. 5B shows early-stage apoptosis (Annexin V+/PI-) induced by oxaliplatin in confluent (GJ formed) $\mathrm{TM}_{3}$ cells. RA (GJIC potentiator) decreased the oxaliplatin-induced apoptotic rate significantly. Notably, in confluent I-10 cells, RA increased the apoptotic rate following oxaliplatin treatment. In confluent $\mathrm{TM}_{3}$ cells, 18- $\alpha-\mathrm{GA}$ and oleamide (GJIC inhibitor) increased the oxaliplatin-induced apoptotic rate significantly. However, 18- $\alpha$-GA and oleamide decreased the apoptotic rate following oxaliplatin treatment in confluent I-10 cells. Thus, the effects of the GJ potentiator/inhibitor on leydig normal/cancer cell apoptosis are similar to the cell survival described above.

Effect of GJIC on the expression of Bcl-2, Bax and caspase-3/9 following oxaliplatin treatment. To determine the possible factor in GJIC-affected apoptosis in the leydig normal and cancer cells treated with oxaliplatin, the apoptotic-associated protein (Bcl-2, Bax and caspase-3/9) was measured with western blot analysis.

Bcl-2 (apoptosis inhibitor) and Bax (apoptosis promoter) are important regulators in the apoptotic pathway. GJIC modulation and oxaliplatin treatment were identical to the results obtained from the Annexin V/PI double staining apoptosis assay. In the leydig normal cells $\mathrm{TM}_{3}$ and the GJIC potentiator RA increased the ratio of $\mathrm{Bcl}-2 / \mathrm{Bax}$. However, $18-\alpha-\mathrm{GA}$ and oleamide (GJIC inhibitor) decreased this ratio (Fig. 6, left panel). The effect of GJIC on Bcl-2/Bax exerted an opposite effect in the leydig cancer I-10 cells. As shown in Fig. 6 (right panel), the ratio of $\mathrm{Bcl}-2 / \mathrm{Bax}$ was decreased in the GJIC potentiator (RA) pretreatment group, but was increased in the GJIIC inhibitor (18- $\alpha$-GA and oleamide) group.

The caspase cascade system is crucial in the induction, transduction and amplification of intracellular apoptotic signals. In the mitochondrial pathway, the release 

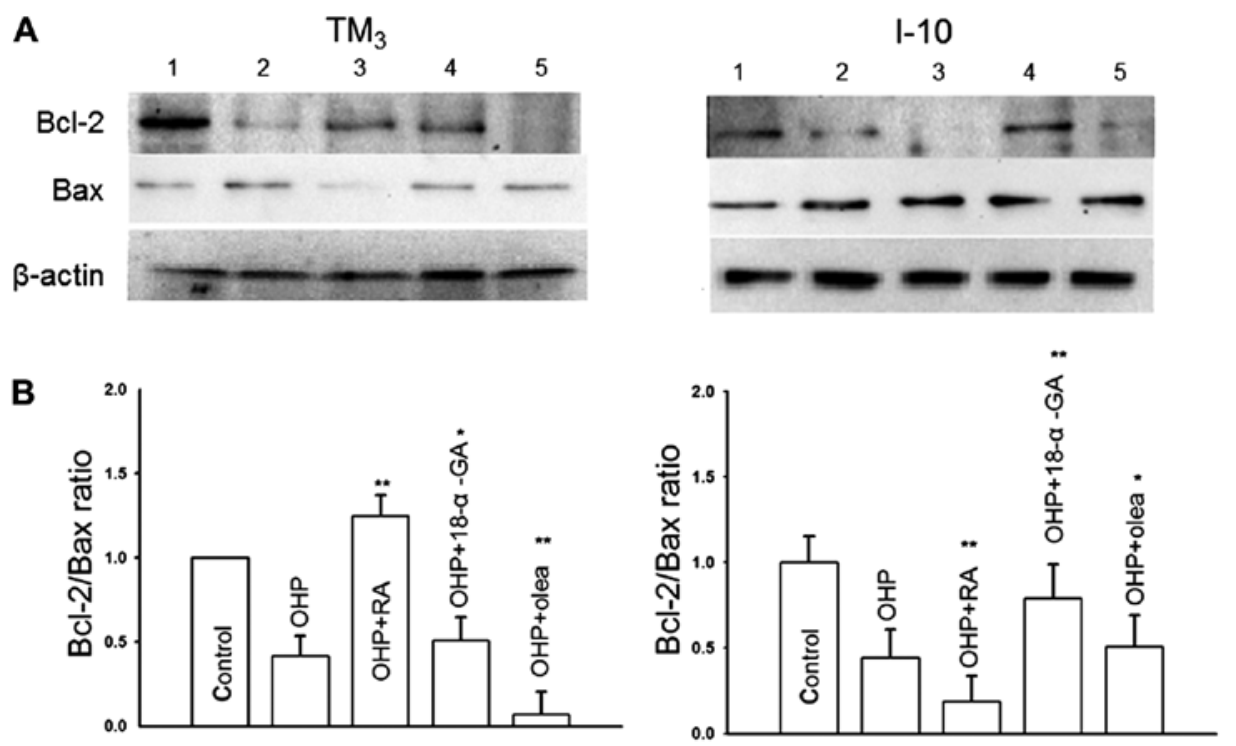

Figure 6. Effect of gap junction on the ratio of Bcl-2/Bax expression treated with oxaliplatin as determined by western blot analysis. (A) Representative bands of Bcl-2 and Bax expression in $\mathrm{TM}_{3}$ cells (left panel) and I-10 cells (right panel) following treatment with oxaliplatin ( $20 \mu \mathrm{M}, 12 \mathrm{~h}$ ). Lanes: 1, control; 2 , oxaliplatin; 3 , oxaliplatin + retinoic acid; 4, oxaliplatin + 18- $\alpha-\mathrm{GA} ; 5$, oxaliplatin + oleamide. (B) Columns are the means from densitometric scanning from blots; bars are the standard deviation $(\mathrm{n}=3) .{ }^{*} \mathrm{P}<0.05$ and ${ }^{* *} \mathrm{P}<0.01$, significantly different from the oxaliplatin group. OHP, oxaliplatin; RA, retinoic acid; Olea, oleamide.
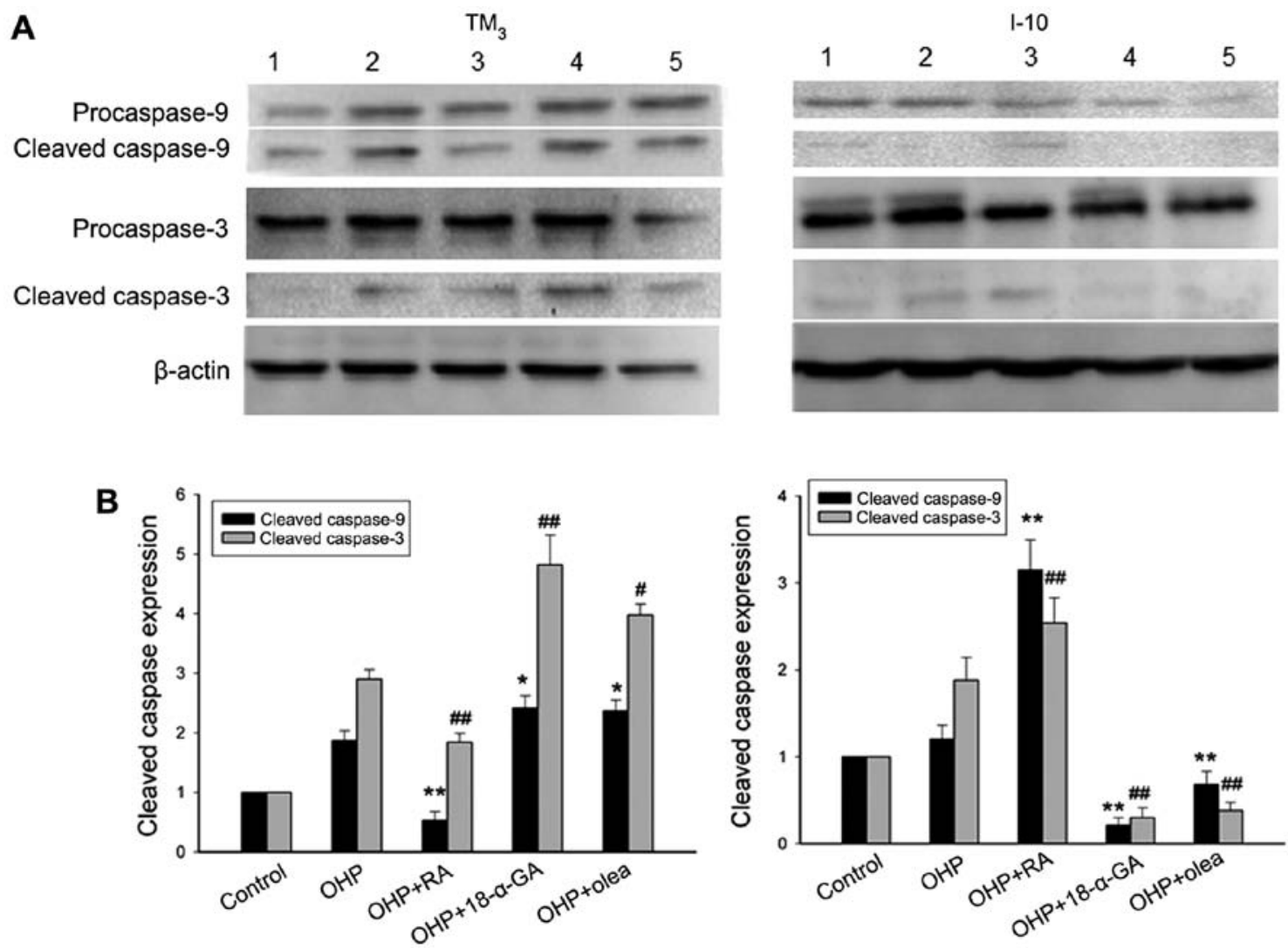

Figure 7. Effect of gap junction on the cleavage of caspase-3 and -9 treated with oxaliplatin as determined by western blot analsysis. (A) Representative bands of caspase- 3 and -9 cleavage in $\mathrm{TM}_{3}$ cells (left panel) and I-10 cells (right panel) folllowing treatment with oxaliplatin ( $\left.20 \mu \mathrm{M}, 12 \mathrm{~h}\right)$. Lanes: 1, control; 2 , oxaliplatin; 3 , oxaliplatin + retinoic acid; 4 , oxaliplatin + 18- $\alpha-\mathrm{GA} ; 5$, oxaliplatin + oleamide. (B) Columns are the means from densitometric scanning from blots; bars are the standard deviation $(\mathrm{n}=3) .{ }^{*} \mathrm{P}<0.05$ and ${ }^{* *} \mathrm{P}<0.01$, significantly different from the oxaliplatin group. OHP, oxaliplatin; RA, retinoic acid; Olea, oleamide.

of cytochrome $c$ from mitochondria activates caspase-9, and downstream, caspase- 3 is subsequently activated. Fig. 7 shows that $20 \mu \mathrm{M}$ oxaliplatin for $12 \mathrm{~h}$ activated the caspase- $3 / 9$ in leydig normal and cancer cells. In normal cells $\mathrm{TM}_{3}$, RA decreased the cleavage of caspase-3/9 following oxaliplatin treatment. However, $18-\alpha-\mathrm{GA}$ and oleamide increased the 
caspase-3/9 cleavage (Fig. 7A, left panel). As shown in Fig. 7 (right panel), caspase-3/9 activation was increased in the RA group, but decreased in the $18-\alpha-\mathrm{GA}$ and oleamide groups. This result suggested that GJIC modulates the mitochondrial pathway in oxaliplatin-induced apoptosis.

\section{Discussion}

The results of the present study show that GJIC composed of Cx43 affected oxaliplatin cytotoxicity conversely in mouse leydig normal and cancer cells. Cell survival, apoptosis, apoptosis regulator $\mathrm{Bax}, \mathrm{Bcl}-2$ and activation of caspase-3/9 were assessed to compare the normal/cancer cell response to oxaliplatin. GJIC was manipulated through pharmacological agents to become upregulated or downregulated. The results indicate that the direction (increased/decreased cell viability/apoptosis) of the GJIC modulation on oxaliplatin cytotoxicity depends on the oncogenic status of cells. In $\mathrm{TM}_{3}$ leydig normal cells, enhancement of GJIC decreased toxicity, whereas inhibition of GJIC increased toxicity. However, in I-10 leydig cancer cells, the toxicity affected through GJIC modulation exhibited an opposite effect.

Oxaliplatin is the third generation platinum drug, which contains diaminocyclohexane (DACH). The activity, pharmacological mechanisms and resistance of oxaliplatin appear to be different from those of the first (e.g., cisplatin) and second generation (e.g., carboplatin) drugs (32). The cytotoxicity of oxaliplatin is thought to inhibit DNA synthesis. The chemical structure of bulky DACH ring in oxaliplatin can result in the formation of platinum-DNA adducts. The intra- and interstrand platinum-DNA-protein cross-links impact on nuclear metabolism and chromatin organization and disrupt DNA replication and repair. Furthermore, oxaliplatin induces apoptosis in a variety of carcinoma cells (testis, colorectum, liver) via different pathways (33). Chater et al (31) showed that oxaliplatin induced an increase of p53 expression and a decrease of inhibitors of apoptosis proteins (IAP) in mouse. Hayward et al (34) reported that Bcl-xL downregulation amplified oxaliplatin-induced apoptosis through the p53- and Bax-mediated apoptotic pathway. Gourdier et al (35) demonstrated oxaliplatin-induced apoptosis via the mitochondrial pathway in human colon cancer cells.

Direct communication between cells, mediated by gap junctions, is considered an indispensable mechanism in the maintenance of cellular homeostasis. Gap junctional intercellular communication is actively involved in virtually all aspects of the cell life cycle, ranging from cell growth to cell death $(36,37)$. Gap junctions have widely been described to modulate cell survival and cell death. Previous reports $(15,16,26)$ have shown that GJIC can enhance the cytotoxicity of antineoplastic drugs in different cell types. Notably, GJIC also reduced the damage following oxidative stress or ischemia in epithelium, astrocyte and neuron. However, the possible mechanism involved in GJIC affecting cell survival and/or death is poorly understood. Hong et al (26) also reported downregulation of GJIC by pharmacological inhibition/siRNA suppression decreased cisplatin cytoxicity in testicular cancer cells (MLTC-1) but enhanced cytoxicity in normal cells $\left(\mathrm{TM}_{3}\right.$ and $\mathrm{TM}_{4}$ ). Additionally, the increased DNA interstrand crosslinks were associated with enhanced toxicity through GJIC downregulation in normal cells. However, those authors did not identify oxalipatin cytotoxicity, especially that inducing apoptosis, following GJIC modulation (inhibition/upregulation). To the best of our knowledge, in the present study, we show for the first time that the GJIC permeable signal affects oxaliplatin-inducing apoptosis conversely in testicular normal and cancer cells. Additionally, we demonstrate that GJIC-mediated opposite effect following treatment with oxaliplatin is associated with Bcl-2/Bax and caspase-3/9.

The involvement of GJIC in the increase of oxaliplatin cytotoxicity in testicular cancer cells suggests that oxaliplatin suppresses cell death directly and generates specific death signals propagated through gap junctions. However, in testicular normal cells, GJIC exerts opposite effects to that in cancer cells. The reason for the protective effect in normal cells is probably that GJIC transmits particular survival signals or inhibits death signals activated when cells are exposed to oxaliplatin. However, the exact death or survival signal properties remain to be elucidated.

The candidate signals that were generated by treatment with oxaliplatin and transmitted through gap junction composed of $\mathrm{Cx} 43$ are probably first or second messengers, such as $\mathrm{Ca}^{2+}$, inositol 1.4.5-triphosphate $\left(\mathrm{IP}_{3}\right), \mathrm{ATP}$ and cAMP. Decrock et al (38) have shown that inhibition of $\mathrm{IP}_{3}$ by antagonizing of the $\mathrm{IP}_{3}$ receptor and $\mathrm{IP}_{3}$-promoted degradation can decrease cytochrome $c$-induced apoptosis. Kameritsch et al (39) reported that the $\mathrm{IP}_{3}$ receptor blocker decreased steptonigrin-induced $\mathrm{Ca}^{2+}$ increase in GJ-coupled HeLa cells transfected with $\mathrm{Cx} 43$. Thus, $\mathrm{IP}_{3}$ is likely the vital death signal that amplifies apoptosis through the functional gap junction.

The Bcl-2 family and caspase-3/9 are known as crucial regulators of intrinsic apoptosis. In the Bcl-2 family, Bcl-2 and Bax exert an opposite effect on apoptosis induction. Bcl-2 is the apoptotic inhibition protein that protects cells from apoptosis by preventing caspase-3-dependent proteolytic cascade (40). However, the apoptosis regulator Bax promotes apoptosis by binding to and antagonizing the $\mathrm{Bcl}-2$ protein. Our results show that, in testicular cancer cells, the upregulation of gap junction decreased the ratio of $\mathrm{Bcl}-2 / \mathrm{Bax}$, while the ratio was increased when GJIC was downregulated. This apoptosis-related protein alteration was converse in testicular normal cells. For the downstream of Bcl-2/Bax, caspase activation was the most important executor for induced apoptosis. The 'mitochondrial' (caspase-9 regulated) and 'death receptor' (caspase-8 regulated) pathways lead to apoptosis. Previously, our results showed that the mitochondrial pathway is involved in the GJIC-amplified apoptosis following cisplatin exposure in HeLa cells expressing Cx26/Cx32 (16). In the present study, after GJIC upregulation, the mitochondrial pathway-related caspase-9 and downstream caspase-3-activated cleavages were enhanced in cancer cells, but impaired in normal cells. Inhibition of GJIC exerts the opposite effect on caspase-3/9 activation in the testicular cancer/normal cells.

Taken together, the present study has demonstrated the GJIC-mediated opposite (toxic or protective) effect on the testicular cancer and normal cells. The important implication is that enhanced GJIC has amplified cytotoxicity in testicular cancer cells, but off-target injury or protection in normal cells. It suggests GJIC modulation is a potential candidate target in testicular cancer chemotherapy. 


\section{Acknowledgements}

The present study was supported by the National Natural Science Foundation of China (grant no. 81001457).

\section{References}

1. Chauvigne F, Zapater C, Gasol JM and Cerda J: Germ-line activation of the luteinizing hormone receptor directly drives spermiogenesis in a nonmammalian vertebrate. Proc Natl Acad Sci USA 111: 1427-1432, 2014.

2. Chauvigne F, Verdura S, Mazon MJ, et al: Follicle-stimulating hormone and luteinizing hormone mediate the androgenic pathway in Leydig cells of an evolutionary advanced teleost. Bio Reprod 87: 35, 2012.

3. Sakaeda T, Kadoyama K and Okuno Y: Adverse event profiles of platinum agents: data mining of the public version of the FDA adverse event reporting system, AERS, and reproducibility of clinical observations. Int J Med Sci 8: 487-491, 2011.

4. Martelli L, Ragazzi E, Di Mario F, Basato M and Martelli M: Cisplatin and oxaliplatin cytotoxic effects in sensitive and cisplatin-resistant human cervical tumor cells: time and mode of application dependency. Anticancer Res 29: 3931-3937, 2009.

5. Perry J, Powles T, Shamash J, et al: The relative activity of cisplatin, oxaliplatin and satraplatin in testicular germ cell tumour sensitive and resistant cell lines. Cancer Chemother Pharmacol 64: 925-933, 2009.

6. Stordal BK, Davey MW and Davey RA: Oxaliplatin induces drug resistance more rapidly than cisplatin in $\mathrm{H} 69$ small cell lung cancer cells. Cancer Chemother Pharmacol 58: 256-265, 2006.

7. Stordal B, Pavlakis N and Davey R: Oxaliplatin for the treatment of cisplatin-resistant cancer: a systematic review. Cancer Treat Rev 33: 347-357, 2007.

8. Tozawa K, Oshima T, Kobayashi T, et al: Oxaliplatin in treatment of the cisplatin-resistant MKN45 cell line of gastric cancer. Anticancer Res 28: 2087-2092, 2008.

9. Montopoli M, Ragazzi E, Froldi G and Caparrotta L: Cell-cycle inhibition and apoptosis induced by curcumin and cisplatin or oxaliplatin in human ovarian carcinoma cells. Cell Prolif 42 : 195-206, 2009.

10. Su V and Lau AF: Connexins: mechanisms regulating protein levels and intercellular communication. FEBS Lett 588 1212-1220, 2014

11. Kjenseth A, Fykerud T, Rivedal E and Leithe E: Regulation of gap junction intercellular communication by the ubiquitin system. Cell Signal 22: 1267-1273, 2010.

12. Kahiri CN, Khalil MW, Tekpetey F and Kidder GM: Leydig cell function in mice lacking connexin43. Reproduction 132: 607-616, 2006

13. Gunther S, Fietz D, Weider K, Bergmann M and Brehm R: Effects of a murine germ cell-specific knockout of Connexin 43 on Connexin expression in testis and fertility. Transgenic Res 22: 631-641, 2013

14. Giese S, Hossain H, Markmann M, et al: Sertoli-cell-specific knockout of connexin 43 leads to multiple alterations in testicular gene expression in prepubertal mice. Dis Model Mech 5: 895-913, 2012.

15. Sato T, Neschadim A, Lavie A, Yanagisawa T and Medin JA: The engineered thymidylate kinase (TMPK)/AZT enzyme-prodrug axis offers efficient bystander cell killing for suicide gene therapy of cancer. PLoS One 8: e78711, 2013.

16. Tong X, Dong S, Yu M, Wang Q and Tao L: Role of heteromeric gap junctions in the cytotoxicity of cisplatin. Toxicology 310: 53-60, 2013.

17. Autsavapromporn N, De Toledo SM, Jay-Gerin JP, Harris AL and Azzam EI: Human cell responses to ionizing radiation are differentially affected by the expressed connexins. J Radiat Res 54: 251-259, 2013

18. Ohshima Y, Tsukimoto M, Harada $\mathrm{H}$ and Kojima S: Involvement of connexin 43 hemichannel in ATP release after gamma-irradiation. J Radiat Res 53: 551-557, 2012.

19. Mancuso M, Pasquali E, Leonardi S, et al: Role of connexin43 and ATP in long-range bystander radiation damage and oncogenesis in vivo. Oncogene 30: 4601-4608, 2011.
20. Colombo BM, Benedetti S, Ottolenghi S, et al: The 'bystander effect': association of U-87 cell death with ganciclovir-mediated apoptosis of nearby cells and lack of effect in athymic mice. Hum Gene Ther 6: 763-772, 1995.

21. Hutnik CM, Pocrnich CE, Liu H, Laird DW and Shao Q: The protective effect of functional connexin 43 channels on a human epithelial cell line exposed to oxidative stress. Invest Ophthalmol Vis Sci 49: 800-806, 2008.

22. Lin D and Takemoto DJ: Oxidative activation of protein kinase Cgamma through the $\mathrm{C} 1$ domain. Effects on gap junctions. J Biol Chem 280: 13682-13693, 2005.

23. Kozoriz MG, Bechberger JF, Bechberger GR, et al: The connexin43 C-terminal region mediates neuroprotection during stroke. J Neuropathol Exp Neurol 69: 196-206, 2010.

24. Giardina SF, Mikami M, Goubaeva F and Yang J: Connexin 43 confers resistance to hydrogen peroxide-mediated apoptosis Biochem Biophys Res Commun 362: 747-752, 2007.

25. Farahani R, Pina-Benabou MH, Kyrozis A, et al: Alterations in metabolism and gap junction expression may determine the role of astrocytes as 'good samaritans' or executioners. Glia 50: 351-361, 2005.

26. Hong X, Wang Q, Yang Y, et al: Gap junctions propagate opposite effects in normal and tumor testicular cells in response to cisplatin. Cancer Lett 317: 165-171, 2012.

27. Koreen IV, Elsayed WA, Liu YJ and Harris AL: Tetracyclineregulated expression enables purification and functional analysis of recombinant connexin channels from mammalian cells. Biochem J 383: 111-119, 2004.

28. Tao L and Harris AL: 2-aminoethoxydiphenyl borate directly inhibits channels composed of connexin 26 and/or connexin32. Mol Pharmacol 71: 570-579, 2007.

29. Wu J, Taylor RN and Sidell N: Retinoic acid regulates gap junction intercellular communication in human endometrial stromal cells through modulation of the phosphorylation status of connexin 43. J Cell Physiol 228: 903-910, 2013.

30. Kim SJ, Park EY, Hwang TK and Kim JC: Therapeutic effects of connexin inhibitors on detrusor overactivity induced by bladder outlet obstruction in rats. Urology 78: 475.e471-477, 2011.

31. Chater S, Amara S, Moussata D, et al: Differential effects of ionizing radiation and platinum-derivative chemotherapy on apoptotic pathways in testicular germ cells. Int J Radiat Biol 83: 269-278, 2007.

32. Bowden NA: Nucleotide excision repair: why is it not used to predict response to platinum-based chemotherapy? Cancer Lett 346: 163-171, 2014

33. Bonetti A, Giuliani J and Muggia F: Targeted agents and oxaliplatin-containing regimens for the treatment of colon cancer. Anticancer Res 34: 423-434, 2014.

34. Hayward RL, Macpherson JS, Cummings J, Monia BP, Smyth JF and Jodrell DI: Enhanced oxaliplatin-induced apoptosis following antisense Bcl-xl down-regulation is p53 and Bax dependent: genetic evidence for specificity of the antisense effect. Mol Cancer Ther 3: 169-178, 2004.

35. Gourdier I, Crabbe L, Andreau K, Pau B and Kroemer G: Oxaliplatin-induced mitochondrial apoptotic response of colon carcinoma cells does not require nuclear DNA. Oncogene 23: 7449-7457, 2004.

36. Gaete PS, Lillo MA and Figueroa XF: Functional role of connexins and pannexins in the interaction between vascular and nervous system. J Cell Physiol 229: 1336-1345, 2014.

37. Carette D, Gilleron J, Chevallier D, Segretain D and Pointis G: Connexin a check-point component of cell apoptosis in normal and physiopathological conditions. Biochimie 101: 1-9, 2013.

38. Decrock E, Krysko DV, Vinken M, et al: Transfer of $\mathrm{IP}_{3}$ through gap junctions is critical, but not sufficient, for the spread of apoptosis. Cell Death Differ 19: 947-957, 2012.

39. Kameritsch P, Khandoga N, Pohl U and Pogoda K: Gap junctional communication promotes apoptosis in a connexintype-dependent manner. Cell Death Dis 4: e584, 2013.

40. Swanton E, Savory P, Cosulich S, Clarke P and Woodman P Bcl-2 regulates a caspase-3/caspase-2 apoptotic cascade in cytosolic extracts. Oncogene 18: 1781-1787, 1999. 